\title{
Topoisomerase Interaction
}

National Cancer Institute

\section{Source}

National Cancer Institute. Topoisomerase Interaction. NCI Thesaurus. Code C40548.

Topoisomerase Interaction involves temporary non-covalent binding through

intermolecular physical forces of attraction and spatial complementarity with enzymes

that change the degree of supercoiling in DNA by cutting one or both strands. Type I

topoisomerases cut only one strand of DNA, while type II topoisomerases cut both strands of DNA. 